\title{
A Survey of Satellite High Resolution Image Classification
}

\author{
Ruby Bharti \\ Scholar \\ Department of Computer \\ Science \& Engg \\ BBAU Lucknow
}

\author{
Jitendra Kurmi \\ Assistant Professor \\ Department of Computer \\ Science \& Engg \\ BBAU Lucknow
}

\begin{abstract}
The aim of this research is to present a detailed step-by-step method for classification of very high resolution urban satellite images (VHRSI) into specific classes such as road, building, vegetation, etc., using fuzzy logic. In this study, object-based image analysis is used for image classification. The main problems in high resolution image classification are the uncertainties in the position of object borders in satellite images and also multiplex resemblance of the segments to different classes. In order to solve this problem, fuzzy logic is used for image classification, since it provides the possibility of image analysis using multiple parameters without requiring inclusion of certain thresholds in the class assignment process Image classification is the most important process in pattern reorganization. Image classification is one of the most complex areas in image processing. It is more complex and difficult to classify if it contain blurry and noisy content. There are several methods to classify images and they provide good classification result but they fail to provide satisfactory classification result when the image contains blurry and noisy content. The two main methods for image classification are supervised and unsupervised classification. In general, this is a final stage of pattern matching. The classification process described the percentage of accuracy in pattern recognition. Feature extraction is another vital stage in pattern matching. These extracted feature are used for classification of the image database, that is pattern matching.
\end{abstract}

\section{General Terms}

Blurry images, Image classification, Noisy images, Supervised classification, Unsupervised classification.

\section{Keywords}

Classification, Pattern recognition, fuzzy rule based systems, very high resolution satellite imagery.

\section{INTRODUCTION}

Image classification is one of the most complex areas in image processing. A machine learning technique is used to assign class label to set of unclassified data. In the classification technique there are two types of classification techniques[1].

In Supervised classification, the some pixels are known grouped and gives the label to classes. This process is known as training. After that classifier uses trained pixels for classify other images.

In Unsupervised classification, pixels are grouped with the help of their properties. This process known as clustering and groups are known a cluster. In this user decide how many clusters he wants. The unsupervised classification used when no trained pixels are available.
With the development of satellite images to provide finer spatial resolutions, they can provide finer more details in urban mapping [2]. However, considering high spectral variation within the same urban class and low spectral variations between different urban classes, image classification has become a challenging issue $[2,3]$.

Considering the existence of extreme level of detail in very high resolution urban satellite images (VHRSI), object-based methods are being increasingly employed for image classification since they have a higher resemblance to human interpretation skills and in object-based image analysis, object characteristics such as shape, texture, topological information, and spectral response can also be used [3,4]. In object-based image classifications, an image is divided into nonoverlapping segments which are then assigned to different classes using specific methods; for example, [5] presented a method for object-based image classification using a neural network. They used a kernel called - cloud basell function for classification. In [6] rule-based classification of very high resolution images, using cartographic data, was used. In the segmentation step, the seeds were picked in the center of cartographic objects; then, using some contextual rules, they classified the image. Although object-based image analysis is not mature enough to be used in automatic image analysis, it is still very promising [5].

Detecting various image objects such as buildings and roads in VHRSI is quite problematic due to uncertain object borders in VHRSI as well as multiplex resemblance of the segments to different classes. Sometimes even the human eye has difficulty differentiating some image objects. Thus, a significant number of studies have added ancillary data, such as road maps, building maps, etc., to the process in order to make the procedure easier [7-10]. Needless to say, those ancillary data are not always available for other studies. In this project, no ancillary data are used. Another group of articles use fuzzy logic to deal with the mentioned complexity Fuzzy logic, which is developed by [11], has been used in image classification in several studies [12-20]. In [12], a fuzzy membership matrix for supervised image classification was used. Introducing partial membership of pixels, mixed pixels could be identified and more accurate classification results could be achieved. In addition, [13] used fuzzy logic for Spot image classification. In [14], fuzzy borders for image segmentation was used. In [15], fuzzy segmentation for object-based image classification was adopted. They used a fuzzy classification method on a segmented image to classify large scale areas such as mining fields and transit sites. In [16], Object-Oriented fuzzy analysis of remote sensing data for GIS-ready information was used. In [17-20], methods for hierarchical image classification using fuzzy logic were also presented. As can be seen fuzzy logic is a quite popular in 
satellite image analysis. Considering the uncertainties in image pixels/segments, a fuzzy inference system can be of great help in image classification.

\section{IMAGE CLASSIFICATION}

Image classification is one of the important and complex processes in image processing. There are several image classification methods. The two main image classification methods are supervised classification and unsupervised classification.

\section{A. Supervised Classification}

Supervised classification requires prior information before testing process and it must collected by analyst. The steps in

supervised classification approach are:

- Training areas for each informational class are identified by analyst

- Signatures identifies(mean, variance, covariance, etc)

- All pixels are classified

- Map Informational Class

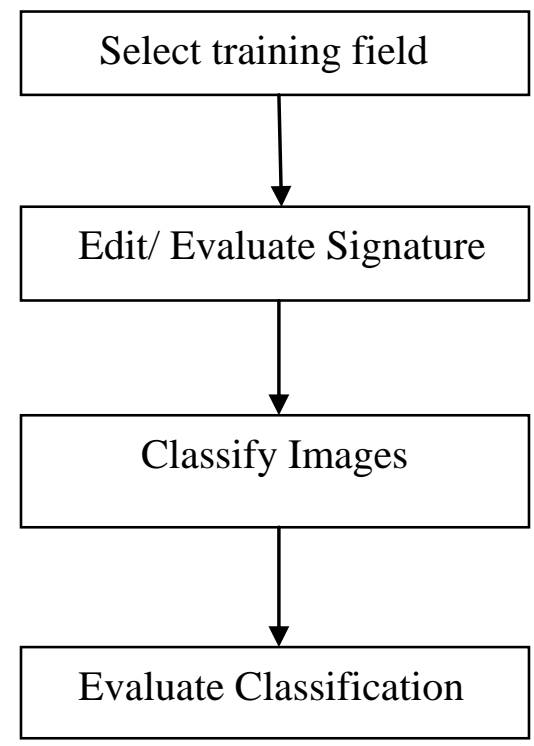

Proposed a new approach for improving performance of object class recognition by combining different features with local features. In this, the features are extracted from the image, which are boundary-based shape features and local features.

\section{B. Unsupervised Classification}

In unsupervised classification, prior information is not needed. It does not require human annotation, it is fully automated. This algorithm identifies clusters in data and also analyst labels clusters. The steps in unsupervised classification are:

- Clustering data

- All pixels are classified based on clusters

- $\quad$ Spectral class map

- Clusters are labeled by analyst

- Map informational class

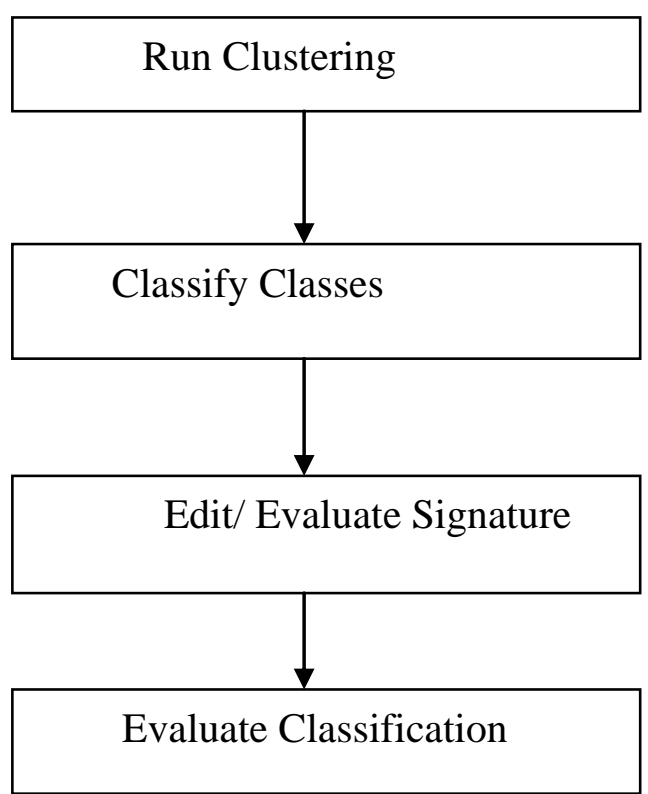

Proposed a new method for classifying outdoor scenes. First, by using one-class classification and patch-based clustering algorithms images are partitioned into regions. Secondly, to obtain region types codebook, the resulting regions thus obtained are clustered, and then two models are constructed for scene representation: a bag of individual regions representation where each region is considered separately, and a bag of region pairs representation this means regions with same spatial relationships are considered together

\section{IMAGE CLASSIFCATION STEPS}

Image Classification includes following steps:

a. Image Acquisition: acquire the images from for image processing.

b. Image Pre-Processing: In preprocessing image transformation, noise removal, atmospherically correction techniques are used.

c. Feature Extraction: Extracting the important characteristics of the image.

Classification: The images are classified based on the extracted features into predefined categories by using suitable methods that compare the image pattern with images which inside the database.

\section{ARCHITECHTURE OF FUZZY}

LOGIC:

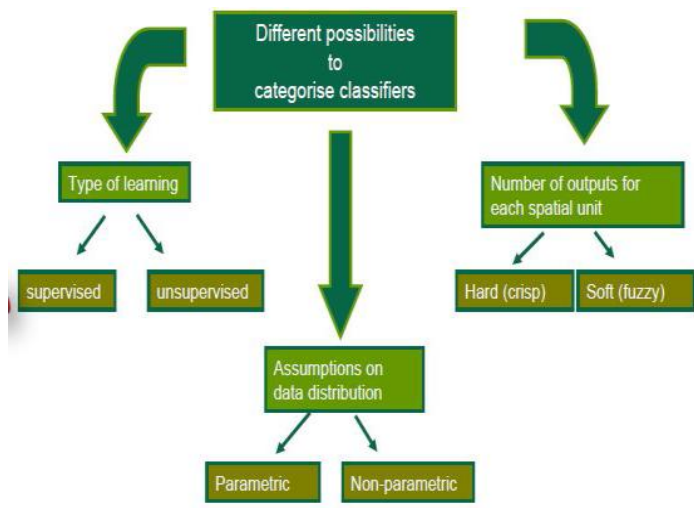




\section{RELATED WORK}

The paper by Jispa kurian et al. [1], the challenge of image classification from a large dataset is the subject of the recent research work. The support vector machine (SVM) classifier shows to be very effective in image classification. data and this method capable of representing AND, OR and NOT. Similarly, Bahram Salehi [3] in 2012 the researched on the objective "Object-Based Classification of Urban Areas Using VHR Imagery and Height Points Ancillary Data " proposed the methodology Land cover classification of very high resolution (VHR) imagery over urban areas is an extremely challenging task. Impervious land covers such as buildings, roads, and parking lots are spectrally too similar to be separated using only the spectral information of VHR imagery. The paper by T. Bauer and K. Steinnocher[4] in 2001 researched on "Per parcel land use classification in urban areas applying a rule based technique " Monitoring urban land use requires very high spatial resolution image data, acquired either by airborne or spaceborne sensor systems. The information content of such images is very complex, thus information extraction is currently performed on the basis of visual interpretation. The aim of the research described in this study is to formalise the visual interpretation procedure in order to automate the land use mapping process for urban areas. conclusion to be drawn from the study is the suitability of IKONOS-data for urban land use mapping, thus representing an alternative to aerial photographs for updating and maintaining cartographic and geographic databases

The paper Mourad Bouziani [7] in 2010 researched on the objective"Rule-Based Classification of a Very High Resolution Image in an Urban Environment Using Multispectra Segmentation Guided by Cartographic Data" Classification algorithms based on single-pixel analysis often do not give the desired result when applied to high-spatial resolution remote-sensing data. In such cases, classification algorithms based on object-oriented image segmentation are needed. There are many segmentation algorithms in the literature, but few have been applied in urban studies to classify a highs patial- resolution remote-sensing image.

A classifier is considering more efficient if they can predict correctly. Image Classification is important to extract the pattern or feature from the available input datasets.

\section{CONCLUSION}

This paper gives the brief knowledge on some supervised classification methods used in Image Classification. Although the accuracies for building and bare land detection in this project are not as high as other classes, they are still higher than those of the check project. In addition, the presented method succeeded in detecting roads, shadows, and vegetation. Referring to the confusion matrix, the accuracy related to these three classes is fairly promising. However, the crisp thresholds for classification did not produce so high accuracies. Keeping in mind that with the current knowledge of object-based methods, building detection is extremely challenging without using ancillary data, hence, offering a comprehensive image classification system which will be applicable to every kind of images is not possible, the presented method can be useful as a sample for other fuzzybased image classification projects. Of course, if there are any other ancillary data available, the produced results will be significantly better. Therefore, in similar projects, the presented specifications for each class can be used and if any additional information is available, it will help to improve the accuracy of the classification.

\section{ACKNOWLEDGEMENT}

We would like to express our gratitude to all those who gave us the possibility to complete this paper.

\section{REFERENCES}

[1] Jipsa Kurian and V.Karunakaran, 2012. A Survey on Image Classification Methods in IJARECE.

[2] Pacifici, F.; Chini, M.; Emery, W.J, 2009. A neural network approach using multi-scale textural metrics from very high-resolution panchromatic imagery for urban land-use classification. Remote Sens. Environ.

[3] Salehi, B.; Zhang, Y.; Zhong, M.; Dey, V. 2012 Objectbased classification of urban areas using vhr imagery and height points ancillary data. Remote Sens.

[4] Bauer, T.; Steinnocher, K. Per-parcel land 2001 use classification in urban areas applying a rule-based technique. GeoBIT/GIS.

[5] Hofmann, P. 2001 Detection Informal Settlements from IKONOS Image Data Using Methods of Object Oriented Image Analysis-An Example from Cape Town (South Africa). In Remote Sensing of Urban. Areas/Fenerkundung in Urbanen. Raumen; Jürgens, $C$., Ed.; Regensburg, Germany.

[6] Rizvi, I.A.; Mohan, B.K. 2011 Object-based image analysis of high-resolution satellite images using modified cloud basis function neural network and probabilistic relaxation labeling process. IEEE T. Geosci. Remote. Sens.

[7] Bouziani, M.; Goita, K.; He, D.C.2010 Rule-based classification of a very high resolution image in an urban environment using multispectral segmentation guided by cartographic data. IEEE T. Geosci. Remote. Sens.

[8] Moskal, M.; Styers, D.M.; Halabisky,2011 M. Monitoring urban tree cover using object-based image analysis and public domain remotely sensed data. Remote Sens.

[9] Thomas, N.; Hendrix, C.; Congalton, R.G. 2003 A comparison of urban mapping methods using highresolution digital imagery. Photogramm. Eng. Remote Sens.

[10] Watanachaturaporn, P.; Arora, M.K.; Varshney, P.K 2008. Multisource classification using support vector machines: An empirical comparison with decision tree and neural network classifiers. Photogramm. Eng. Remote Sens.

[11] Salehi, B.; Zhang, Y.; Zhong, M.; Dey, V. 2012 A review of the effectiveness of spatial information used in urban land cover classification of VHR imagery. Int. J. Geo Inf.

[12] Wang, F.1990 Fuzzy supervised classification of remote sensing images. IEEE T. Geosci. Remote. Sens.

[13] Nedeljkovic, I. 2006 Image classification based on fuzzy logic. Remote Sens. Spat. Inf. Sci.

[14] Lizarazo, I.; Elsner, P.2009 Fuzzy segmentation for object-based image classification. Int. J. Remote Sens.

[15] Jianxin Wu, 2012 Efficient Hik SVM Learning For Image Classification, IEEE Transactions On Image Processing. 